\title{
A new result on stability analysis for discrete system with interval time-varying delays
}

\author{
Daixi Liao ${ }^{1,2^{*}}\left(\mathbb{D}\right.$, Shouming Zhong ${ }^{1}$, Jun Cheng $^{3 *}$, Can Zhao ${ }^{1}$, Xiaojun Zhang ${ }^{1}$ and Yongbin Yu ${ }^{4}$
}

"Correspondence:

liaodaixizaici@163.com;

jcheng6819@126.com

'School of Mathematical Sciences,

University of Electronic Science and

Technology of China, Chengdu

P.R. China

${ }^{3}$ School of Automation and

Electronic Engineering, Qingdao University of Science and

Technology, Qingdao, P.R. China

Full list of author information is

available at the end of the article

\begin{abstract}
This paper is concerned with the stability criteria for a discrete-time linear system with interval time-varying delays. By using the time delay division we construct an augmented Lyapunov-Krasovskii functional for two delay subintervals. Moreover, we use a new summation inequality to estimate the derivatives of LKFs more accurately and derive less conservative criteria. Finally, we present two numerical examples to demonstrate that the obtained results are less conservative.
\end{abstract}

Keywords: Discrete system; Stability; Lyapunov-Krasovskii functional; Interval time-varying delay

\section{Introduction}

During the transmission and processing of signal or data, time delay inevitably occurs in such practical systems as neural networks and networked control systems. It is well known that the presence of time delay often affects the dynamic behavior of the system, resulting in deterioration or even instability in system performance. Therefore, the research on the stability of time delay systems has been the focus of many scholars [1-5].

One essential research focus on a system with time delay is how to reduce the conservatism of the stability condition. In view of this, many researchers choose to approach this from two perspectives, selecting an appropriate Lyapunov-Krasovskii functional (LKF) and making a more accurate estimation of its derivative. With regard to the former aspect, many significant results have been achieved such as constructing an amplified LKF [6], increasing its integral multiplicity [7], or dividing the time delay interval [8]. With regard to the latter, the Jensen inequality $[9,10]$ is the first powerful tool used to estimate the derivative of LKF in the stability theory of time-delay systems. Later on, the Wirtinger-based integral inequality [11, 12], the free-matrix-based integral inequality [13-15], and a series of integral inequalities [16] have been proposed. These inequalities are widely applied in time delay systems [17-28]. Of course, there are other methods or combinations of different methods, such as the free-weighting matrix approach proposed in the literature [29], the basic idea is introducing some free weighting matrix through the Newton-Leibniz formula and/or the equation of state of the system, and the less conservative stability results can also be obtained.

It is worth noting that not only continuous-time systems, but also discrete-time systems play an important role and have received extensive attention in the literature [30-47]. In 
[12], by using the Wirtinger-based inequality an improved stability condition is obtained. However, the allowable delay upper bound can be further lifted. This inspires our research in this paper.

Based on the above discussions, by means of a new summation inequality and time delay division, less conservative criteria than some existing ones are obtained, and two examples are presented to demonstrate the effectiveness and superiority of the proposed approach. The main contributions of this paper are as follows:

(i) A new summation inequality is obtained by the Schmidt orthogonalization method, and the corresponding result is less conservative.

(ii) The proof of Corollary 1 in [15] can be simplified by the substitution of variables, which is demonstrated by Lemma 2.1 in this paper.

Notation: Throughout this paper, $\Re^{n}$ denotes the $n$-dimensional Euclidean space, and $\Re^{n \times m}$ is the set of all $n \times m$ real matrices; $I$ is used to denote the identity matrix of proper dimension; $\operatorname{diag}\{\cdots\}$ denotes block diagonal matrix, and $\langle f, g\rangle$ denotes the inner product of $f$ and $g ; P>0(\geq 0)$ means that $P$ is a real symmetric and positive definite (semipositive definite) matrix; the symmetric term in a matrix is denoted by $*$, and $\operatorname{sym}[A]=A+A^{T}$; $N^{+}(N)$ is the set of all positive (nonnegative) integers.

\section{Preliminaries}

Consider the following discrete time-varying delay system:

$$
\left\{\begin{array}{l}
x(k+1)=A x(k)+B x(k-d(k)), \quad k \in N, \\
x(k)=\phi(k), \quad k=-d_{2},-d_{2}+1, \ldots, 0,
\end{array}\right.
$$

where $x(k)=\left(x_{1}(k), x_{2}(k), \ldots, x_{n}(k)\right)^{T} \in \Re^{n}$ is the state vector, $\phi(k) \in \Re^{n}$ is the initial condition, $n \in N^{+}, A$ and $B \in \Re^{n \times n}$ are constant matrices, and $d(k)$ is the discrete time-varying delay satisfying $d_{1} \leq d(k) \leq d_{2}$, where $d_{1}$ and $d_{2}$ are nonnegative integers.

Lemma 2.1 Let $x(i)$ be a sequence of discrete-time variables in $[a, a+n] \rightarrow \Re^{n}$. For symmetric matrices $R \in \Re^{n \times n}$ and $Z_{11}, Z_{22}, Z_{33} \in \Re^{4 n \times 4 n}$ and for any matrices $Z_{12}, Z_{13}, Z_{23} \in \Re^{4 n \times 4 n}$ and $N_{1}, N_{2}, N_{3} \in \Re^{4 n \times n}$ satisfying

$$
\Xi=\left(\begin{array}{cccc}
Z_{11} & Z_{12} & Z_{13} & N_{1} \\
* & Z_{22} & Z_{23} & N_{2} \\
* & * & Z_{33} & N_{3} \\
* & * & * & R
\end{array}\right) \geq 0,
$$

we have the following inequality:

$$
-\sum_{i=a}^{a+n-1} \eta^{T}(i) R \eta(i) \leq \omega^{T} \Omega \omega,
$$

where

$$
\eta(i)=x(i+1)-x(i),
$$




$$
\begin{aligned}
& \omega=\left(x^{T}(a+n), x^{T}(a), \frac{1}{n+1} \sum_{i=a}^{a+n} x^{T}(i), \frac{2}{(n+1)(n+2)} \sum_{i=a}^{a+n} \sum_{j=i}^{a+n} x^{T}(j)\right)^{T}, \\
& \Omega=n\left(Z_{11}+\frac{1}{3} Z_{22}+\frac{1}{5} Z_{33}\right)+\operatorname{sym}\left[N_{1} \Pi_{1}+N_{2} \Pi_{2}+N_{3} \Pi_{3}\right], \\
& \Pi_{1}=\varepsilon_{1}-\varepsilon_{2}, \quad \Pi_{2}=\varepsilon_{1}+\varepsilon_{2}-2 \varepsilon_{3}, \quad \Pi_{3}=\varepsilon_{1}-\varepsilon_{2}+6 \varepsilon_{3}-6 \varepsilon_{4}, \\
& \varepsilon_{1}=\left(\begin{array}{llll}
I & 0 & 0 & 0
\end{array}\right), \quad \varepsilon_{2}=\left(\begin{array}{llll}
0 & I & 0 & 0
\end{array}\right), \\
& \varepsilon_{3}=\left(\begin{array}{llll}
0 & 0 & I & 0
\end{array}\right), \quad \varepsilon_{4}=\left(\begin{array}{llll}
0 & 0 & 0 & I
\end{array}\right) .
\end{aligned}
$$

Proof Define

$$
f_{0}(i)=1, \quad f_{1}(i)=i, \quad f_{2}(i)=i^{2} .
$$

By the Schmidt orthogonalization method, where $\langle f, g\rangle=\sum_{i=1}^{n} f(i) g(i)$, we obtain the following orthogonal polynomials $g_{k}(i), k=0,1,2$ :

$$
g_{0}(i)=1, \quad g_{1}(i)=i-\frac{n+1}{2}, \quad g_{2}(i)=i^{2}-(n+1) i+\frac{(n+1)(n+2)}{6} .
$$

Then converting the coefficient of constant term to 1 or -1 by multiplying by a positive number, we have

$$
h_{0}(i)=1, \quad h_{1}(i)=-1+\frac{2}{n+1} i, \quad h_{2}(i)=1-\frac{6}{n+2} i+\frac{6}{(n+1)(n+2)} i^{2},
$$

and $h_{k}(i), k=0,1,2$, satisfy

$$
\left\langle h_{0}, h_{0}\right\rangle=n, \quad\left\langle h_{1}, h_{1}\right\rangle=n \frac{n-1}{3(n+1)}, \quad\left\langle h_{2}, h_{2}\right\rangle=n \frac{(n-1)(n-2)}{5(n+1)(n+2)} .
$$

Let $\varsigma(i)=\left(h_{0}(i) u^{T}, h_{1}(i) u^{T}, h_{2}(i) u^{T}, \xi^{T}(i)\right)^{T}$, where $u=\left(y^{T}(n+1), y^{T}(1), \frac{1}{n+1} \sum_{i=1}^{n+1} y^{T}(i)\right.$, $\left.\frac{2}{(n+1)(n+2)} \sum_{i=1}^{n+1} \sum_{j=i}^{n+1} y^{T}(j)\right)^{T}, y(i)$ is a sequence of discrete-time variable in $[1,1+n] \rightarrow \Re^{n}$, and $\xi(i)=y(i+1)-y(i)$. Noticing that $\sum_{i=1}^{n+1} i y(i)=\sum_{i=1}^{n+1} \sum_{j=i}^{n+1} y(j)$, we can obtain

$$
\sum_{i=1}^{n} h_{k}(i) \xi(i)=\Pi_{k+1} u, \quad k=0,1,2 .
$$

It is easy to see that $\varsigma^{T}(i) \Xi_{\zeta}(i) \geq 0$. Then summing both sides of the inequality before from 1 to $n$ yields

$$
-\sum_{i=1}^{n} \xi^{T}(i) R \xi(i) \leq u^{T} \tilde{\Omega} u
$$

where $\tilde{\Omega}=n\left(Z_{11}+\frac{n-1}{3(n+1)} Z_{22}+\frac{(n-1)(n-2)}{5(n+1)(n+2)} Z_{33}\right)+\operatorname{sym}\left[N_{1} \Pi_{1}+N_{2} \Pi_{2}+N_{3} \Pi_{3}\right]$.

Let $x(i)=y(i-a+1)$ be a sequence of discrete-time variables in $[a, a+n] \rightarrow \Re^{n}$ satisfying

$$
-\sum_{i=a}^{a+n-1} \eta^{T}(i) R \eta(i)=-\sum_{i=1}^{n} \xi^{T}(i) R \xi(i) \leq u^{T} \tilde{\Omega} u=\omega^{T} \tilde{\Omega} \omega .
$$


Table 1 Allowable delay upper bound $d_{2}$ for various $d_{1}$ for Example 1

\begin{tabular}{|c|c|c|c|c|c|c|c|c|c|}
\hline Method & $d_{1}$ & 2 & 4 & 6 & 10 & 15 & 20 & 25 & 30 \\
\hline [32] & $d_{2}$ & 13 & 13 & 14 & 15 & 18 & 22 & 26 & 30 \\
\hline [33] & $d_{2}$ & 13 & 13 & 14 & 17 & 20 & 24 & 29 & 33 \\
\hline [20] & $d_{2}$ & 14 & 15 & 16 & 18 & 21 & 25 & 30 & 34 \\
\hline$[21]$ & $d_{2}$ & 17 & 17 & 18 & 20 & 23 & 27 & 31 & 35 \\
\hline [34] & $d_{2}$ & 18 & 18 & 19 & 20 & 23 & 26 & 30 & 35 \\
\hline$[40]$ & $d_{2}$ & 20 & 21 & 21 & 22 & 24 & 27 & 29 & 34 \\
\hline [43] (Theorem 2) & $d_{2}$ & 22 & 22 & 22 & 23 & 25 & 28 & 32 & 36 \\
\hline [12] (Remark 4) & $d_{2}$ & 20 & 21 & 21 & 23 & 25 & 29 & 32 & 36 \\
\hline [12] (Theorem 1) & $d_{2}$ & 22 & 22 & 22 & 23 & 26 & 29 & 32 & 36 \\
\hline Remark 3 & $d_{2}$ & 21 & 22 & 22 & 23 & 25 & 29 & 32 & 36 \\
\hline Theorem 3.1 & $d_{2}$ & 22 & 22 & 22 & 23 & 26 & 29 & 32 & 36 \\
\hline
\end{tabular}

Table 2 Allowable delay upper bound $d_{2}$ for various $d_{1}$ for Example 2

\begin{tabular}{llrrlll}
\hline Method & $d_{1}$ & 2 & 5 & 7 & 10 & 20 \\
\hline$[33]$ & $d_{2}$ & 7 & 9 & 11 & 14 & 24 \\
{$[21]$} & $d_{2}$ & 9 & 11 & 13 & 16 & 26 \\
{$[40]$} & $d_{2}$ & 13 & 14 & 16 & 19 & 29 \\
{$[12]$ (Theorem 1) } & $d_{2}$ & 14 & 17 & 18 & 21 & 31 \\
Theorem 3.1 & $d_{2}$ & 15 & 17 & 18 & 21 & 31 \\
\hline
\end{tabular}

Define $\Omega=\tilde{\Omega}+\frac{2 n}{3(n+1)} Z_{22}+\frac{6 n^{2}}{5(n+1)(n+2)} Z_{33}$. Then $\Omega$ is affinely dependent on $n$, and we obtain (2). This completes the proof.

Remark 1 Define $f_{3}(i)=i^{3}$. Then $h_{3}(i)=-1+\lambda \frac{12}{n+3} i-\frac{30}{(n+2)(n+3)} i^{2}+\frac{20}{(n+1)(n+2)(n+3)} i^{3}$, where $\lambda=\frac{6 n^{2}+15 n+11}{6(n+1)(n+2)}$, and it is not the fourth Legendre polynomial anymore [16]. Thus deriving a general form of the discrete inequality (2) is still an interesting and challenging task.

Remark 2 By the substitution of variables demonstrated in Lemma 2.1, the proof of Corollary 1 in [15] can be simplified. By means of the new summation inequality the results calculated based on the criteria given in this paper are less conservative than those reported in the existing literature from Tables 1 and 2.

\section{Main results}

For reducing the conservatism, we further present a delay partitioning method, where the time delay interval is divided into two segments: $\left[d_{1}, d_{2}\right]=\left[d_{1}, d\right] \cup\left[d, d_{2}\right]$.

Theorem 3.1 For given positive integers $d_{1}$ and $d_{2}$ satisfying $d_{1} \leq d(k) \leq d_{2}$, system (1) is asymptotically stable if there exist matrices $P>0\left(\in \mathfrak{R}^{4 n \times 4 n}\right), Q_{i}>0\left(\in \mathfrak{R}^{n \times n}\right)$, symmetric matrices $R \in \Re^{n \times n}$ and $Z_{j 11}, Z_{j 22}, Z_{j 33} \in \Re^{4 n \times 4 n}$, any matrices $Z_{j 12}, Z_{j 13}, Z_{j 23} \in \Re^{4 n \times 4 n}$ and $N_{j i} \in \mathfrak{R}^{4 n \times n}(i=1,2,3 ; j=1,2, \ldots, 7)$ such that the following LMIs hold:

$$
\begin{aligned}
\Psi_{1}(d(k)) & =D_{1}+D_{3}+D_{4}+D_{5}+D_{6}+G_{1}(d(k))+G_{3}(d(k))+G_{4}(d(k))+H_{1}+H_{2} \\
& <0, \quad d(k) \in\left[d_{1}, d\right] \cap N, \\
\Psi_{2}(d(k)) & =D_{2}+D_{3}+D_{4}+D_{7}+D_{8}+G_{2}(d(k))+G_{5}(d(k))+G_{6}(d(k))+H_{1}+H_{3} \\
& <0, \quad d(k) \in\left[d, d_{2}\right] \cap N,
\end{aligned}
$$




$$
\Xi_{j}=\left(\begin{array}{cccc}
Z_{j 11} & Z_{j 12} & Z_{j 13} & N_{j 1} \\
* & Z_{j 22} & Z_{j 23} & N_{j 2} \\
* & * & Z_{j 33} & N_{j 3} \\
* & * & * & R_{i}
\end{array}\right) \geq 0, \quad \text { and } \quad i= \begin{cases}1 & \text { if } j=1, \\
2 & \text { if } j=2,3,5, \\
3 & \text { if } j=4,6,7,\end{cases}
$$

where

$$
\begin{aligned}
& D_{1}=E_{2}^{T} P E_{2}-E_{1}^{T} P E_{1} \text {, } \\
& D_{2}=E_{4}^{T} P E_{4}-E_{3}^{T} P E_{3} \text {, } \\
& D_{3}=e_{1}^{T} Q_{1} e_{1}-e_{2}^{T}\left(Q_{1}-Q_{2}\right) e_{2}-e_{4}^{T}\left(Q_{2}-Q_{3}\right) e_{4}-e_{5}^{T} Q_{3} e_{5} \text {, } \\
& D_{4}=d_{1}\left(e_{0}-e_{1}\right)^{T} R_{1}\left(e_{0}-e_{1}\right)+\alpha_{1} \operatorname{sym}\left[N_{11} \Pi_{1}+N_{12} \Pi_{2}+N_{13} \Pi_{3}\right] \alpha_{1}^{T}, \\
& D_{5}=\left(d-d_{1}\right)\left(e_{0}-e_{1}\right)^{T} R_{2}\left(e_{0}-e_{1}\right)+\alpha_{2} \operatorname{sym}\left[N_{21} \Pi_{1}+N_{22} \Pi_{2}+N_{23} \Pi_{3}\right] \alpha_{2}^{T} \\
& +\alpha_{3} \operatorname{sym}\left[N_{31} \Pi_{1}+N_{32} \Pi_{2}+N_{33} \Pi_{3}\right] \alpha_{3}^{T}, \\
& D_{6}=\left(d_{2}-d\right)\left(e_{0}-e_{1}\right)^{T} R_{3}\left(e_{0}-e_{1}\right)+\alpha_{4} \operatorname{sym}\left[N_{41} \Pi_{1}+N_{42} \Pi_{2}+N_{43} \Pi_{3}\right] \alpha_{4}^{T} \text {, } \\
& D_{7}=\left(d-d_{1}\right)\left(e_{0}-e_{1}\right)^{T} R_{2}\left(e_{0}-e_{1}\right)+\alpha_{5} \operatorname{sym}\left[N_{51} \Pi_{1}+N_{52} \Pi_{2}+N_{53} \Pi_{3}\right] \alpha_{5}^{T} \text {, } \\
& D_{8}=\left(d_{2}-d\right)\left(e_{0}-e_{1}\right)^{T} R_{3}\left(e_{0}-e_{1}\right)+\alpha_{6} \operatorname{sym}\left[N_{61} \Pi_{1}+N_{62} \Pi_{2}+N_{63} \Pi_{3}\right] \alpha_{6}^{T} \\
& +\alpha_{7} \operatorname{sym}\left[N_{71} \Pi_{1}+N_{72} \Pi_{2}+N_{73} \Pi_{3}\right] \alpha_{7}^{T}, \\
& G_{1}(d(k))=\operatorname{sym}\left[\left(E_{2}-E_{1}\right)^{T} P F_{1}(d(k))\right], \\
& G_{2}(d(k))=\operatorname{sym}\left[\left(E_{4}-E_{3}\right)^{T} P F_{2}(d(k))\right], \\
& G_{3}(d(k))=\alpha_{2}\left[\left(d(k)-d_{1}\right)\left(Z_{211}+\frac{1}{3} Z_{222}+\frac{1}{5} Z_{233}\right)\right] \alpha_{2}^{T}, \\
& G_{4}(d(k))=\alpha_{3}\left[(d-d(k))\left(Z_{311}+\frac{1}{3} Z_{322}+\frac{1}{5} Z_{333}\right)\right] \alpha_{3}^{T}, \\
& G_{5}(d(k))=\alpha_{6}\left[(d(k)-d)\left(Z_{611}+\frac{1}{3} Z_{622}+\frac{1}{5} Z_{633}\right)\right] \alpha_{6}^{T}, \\
& G_{6}(d(k))=\alpha_{7}\left[\left(d_{2}-d(k)\right)\left(Z_{711}+\frac{1}{3} Z_{722}+\frac{1}{5} Z_{733}\right)\right] \alpha_{7}^{T}, \\
& F_{1}(d(k))=\left(0,0,\left(d(k)-d_{1}+1\right) e_{7}^{T}+(d-d(k)+1) e_{8}^{T}, 0\right)^{T}, \\
& F_{2}(d(k))=\left(0,0,(d(k)-d+1) e_{8}^{T}+\left(d_{2}-d(k)+1\right) e_{9}^{T}, 0\right)^{T}, \\
& E_{1}=\left(e_{1}^{T},\left(d_{1}+1\right) e_{6}^{T}-e_{1}^{T},\left(d_{2}-d+1\right) e_{9}^{T}-e_{2}^{T}-e_{3}^{T}-e_{4}^{T},\right. \\
& \left.\frac{1}{2}\left(d_{1}+1\right)\left(d_{1}+2\right) e_{10}^{T}-\left(d_{1}+1\right) e_{1}^{T}\right)^{T}, \\
& E_{2}=\left(e_{0}^{T},\left(d_{1}+1\right) e_{6}^{T}-e_{2}^{T},\left(d_{2}-d+1\right) e_{9}^{T}-e_{3}^{T}-e_{4}^{T}-e_{5}^{T},\right. \\
& \left.\frac{1}{2}\left(d_{1}+1\right)\left(d_{1}+2\right) e_{10}^{T}-\left(d_{1}+1\right) e_{6}^{T}\right)^{T}
\end{aligned}
$$




$$
\begin{aligned}
E_{3}= & \left(e_{1}^{T},\left(d_{1}+1\right) e_{6}^{T}-e_{1}^{T},\left(d-d_{1}+1\right) e_{7}^{T}-e_{2}^{T}-e_{3}^{T}-e_{4}^{T},\right. \\
& \left.\frac{1}{2}\left(d_{1}+1\right)\left(d_{1}+2\right) e_{10}^{T}-\left(d_{1}+1\right) e_{1}^{T}\right)^{T}, \\
E_{4}= & \left(e_{0}^{T},\left(d_{1}+1\right) e_{6}^{T}-e_{2}^{T},\left(d-d_{1}+1\right) e_{7}^{T}-e_{3}^{T}-e_{4}^{T}-e_{5}^{T},\right. \\
& \left.\frac{1}{2}\left(d_{1}+1\right)\left(d_{1}+2\right) e_{10}^{T}-\left(d_{1}+1\right) e_{6}^{T}\right)^{T}, \\
H_{1}= & \alpha_{1}\left[d_{1}\left(Z_{111}+\frac{1}{3} Z_{122}+\frac{1}{5} Z_{133}\right)\right] \alpha_{1}^{T}, \\
H_{2}= & \alpha_{4}\left[\left(d_{2}-d\right)\left(Z_{411}+\frac{1}{3} Z_{422}+\frac{1}{5} Z_{433}\right)\right] \alpha_{4}^{T}, \\
H_{3}= & \alpha_{5}\left[\left(d-d_{1}\right)\left(Z_{511}+\frac{1}{3} Z_{522}+\frac{1}{5} Z_{533}\right)\right] \alpha_{5}^{T},
\end{aligned}
$$

and

$$
\begin{aligned}
& \alpha_{1}=\left(e_{1}^{T}, e_{2}^{T}, e_{6}^{T}, e_{10}^{T}\right), \quad \alpha_{2}=\left(e_{2}^{T}, e_{3}^{T}, e_{7}^{T}, e_{11}^{T}\right), \quad \alpha_{3}=\left(e_{3}^{T}, e_{4}^{T}, e_{8}^{T}, e_{12}^{T}\right), \\
& \alpha_{4}=\left(e_{4}^{T}, e_{5}^{T}, e_{9}^{T}, e_{13}^{T}\right), \quad \alpha_{5}=\left(e_{2}^{T}, e_{4}^{T}, e_{7}^{T}, e_{11}^{T}\right), \quad \alpha_{6}=\left(e_{4}^{T}, e_{3}^{T}, e_{8}^{T}, e_{12}^{T}\right), \\
& \alpha_{7}=\left(e_{3}^{T}, e_{5}^{T}, e_{9}^{T}, e_{13}^{T}\right), \quad e_{i}=\left(0_{n \times(i-1) n}, I, 0_{n \times(13-i) n}\right), \quad i=1,2, \ldots, 13, \\
& e_{0}=A e_{1}+B e_{3} \text {. }
\end{aligned}
$$

Proof Construct a Lyapunov functional as follows:

$$
V(k)=\sum_{i=1}^{5} V_{i}(k)
$$

where

$$
\begin{aligned}
V_{1}(k)= & \theta^{T}(k) P \theta(k), \\
V_{2}(k)= & \sum_{i=k-d_{1}}^{k-1} x^{T}(i) Q_{1} x(i)+\sum_{i=k-d}^{k-d_{1}-1} x^{T}(i) Q_{2} x(i)+\sum_{i=k-d_{2}}^{k-d-1} x^{T}(i) Q_{3} x(i), \\
V_{3}(k)= & \sum_{i=-d_{1}}^{-1} \sum_{j=k+i}^{k-1} \eta^{T}(j) R_{1} \eta(j), \\
V_{4}(k)= & \sum_{i=-d}^{-d_{1}-1} \sum_{j=k+i}^{k-1} \eta^{T}(j) R_{2} \eta(j), \\
& -d-1 \\
V_{5}(k)= & \sum_{i=-d_{2}}^{k-1} \sum_{j=k+i} \eta^{T}(j) R_{3} \eta(j),
\end{aligned}
$$

and

$$
\theta(k)=\left(x^{T}(k), \sum_{i=k-d_{1}}^{k-1} x^{T}(i), \sum_{i=k-d_{2}}^{k-d_{1}-1} x^{T}(i), \sum_{i=k-d_{1}}^{k-1} \sum_{j=i}^{k-1} x^{T}(j)\right)^{T} .
$$


For simplicity, we first define the following vectors:

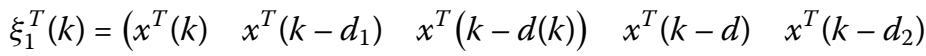

$$
\begin{aligned}
& \left.\begin{array}{llllllll}
U_{1}^{T} & U_{2}^{T} & U_{3}^{T} & U_{4}^{T} & W_{1}^{T} & W_{2}^{T} & W_{3}^{T} & W_{4}^{T}
\end{array}\right), \\
& \xi_{2}^{T}(k)=\left(\begin{array}{lllll}
x^{T}(k) & x^{T}\left(k-d_{1}\right) & x^{T}(k-d(k)) & x^{T}(k-d) & x^{T}\left(k-d_{2}\right)
\end{array}\right. \\
& \begin{array}{llllllll}
U_{1}^{T} & U_{5}^{T} & U_{6}^{T} & U_{7}^{T} & W_{1}^{T} & W_{5}^{T} & W_{6}^{T} & \left.W_{7}^{T}\right),
\end{array}
\end{aligned}
$$

where

$$
\begin{aligned}
& U_{1}=\frac{1}{d_{1}+1} \sum_{i=k-d_{1}}^{k} x(i) \\
& U_{2}=\frac{1}{d(k)-d_{1}+1} \sum_{i=k-d(k)}^{k-d_{1}} x(i) \text {, } \\
& U_{3}=\frac{1}{d-d(k)+1} \sum_{i=k-d}^{k-d(k)} x(i), \\
& U_{4}=\frac{1}{d_{2}-d+1} \sum_{i=k-d_{2}}^{k-d} x(i) \\
& U_{5}=\frac{1}{d-d_{1}+1} \sum_{i=k-d}^{k-d_{1}} x(i) \text {, } \\
& U_{6}=\frac{1}{d(k)-d+1} \sum_{i=k-d(k)}^{k-d} x(i) \text {, } \\
& U_{7}=\frac{1}{d_{2}-d(k)+1} \sum_{i=k-d_{2}}^{k-d(k)} x(i) \text {, } \\
& W_{1}=\frac{2}{\left(d_{1}+1\right)\left(d_{1}+2\right)} \sum_{i=k-d_{1}}^{k} \sum_{j=i}^{k} x(j) \text {, } \\
& W_{2}=\frac{2}{\left(d(k)-d_{1}+1\right)\left(d(k)-d_{1}+2\right)} \sum_{i=k-d(k)}^{k-d_{1}} \sum_{j=i}^{k-d_{1}} x(j) \text {, } \\
& W_{3}=\frac{2}{(d-d(k)+1)(d-d(k)+2)} \sum_{i=k-d}^{k-d(k)} \sum_{j=i}^{k-d(k)} x(j), \\
& W_{4}=\frac{2}{\left(d_{2}-d+1\right)\left(d_{2}-d+2\right)} \sum_{i=k-d_{2}}^{k-d} \sum_{j=i}^{k-d} x(j), \\
& W_{5}=\frac{2}{\left(d-d_{1}+1\right)\left(d-d_{1}+2\right)} \sum_{i=k-d}^{k-d_{1}} \sum_{j=i}^{k-d_{1}} x(j), \\
& W_{6}=\frac{2}{(d(k)-d+1)(d(k)-d+2)} \sum_{i=k-d(k)}^{k-d} \sum_{j=i}^{k-d} x(j) \text {, } \\
& W_{7}=\frac{2}{\left(d_{2}-d(k)+1\right)\left(d_{2}-d(k)+2\right)} \sum_{i=k-d_{2}}^{k-d(k)} \sum_{j=i}^{k-d(k)} x(j) \text {. }
\end{aligned}
$$


Case (i): $d(k) \in\left[d_{1}, d\right] \cap N$. The forward difference of $V_{i}(k)(i=1,2, \ldots, 5)$ is given by

$$
\begin{aligned}
\triangle V_{1}(k)= & \theta^{T}(k+1) P \theta(k+1)-\theta^{T}(k) P \theta(k) \\
= & \xi_{1}^{T}(k)\left\{E_{2}^{T} P E_{2}-E_{1}^{T} P E_{1}+\operatorname{sym}\left[\left(E_{2}-E_{1}\right)^{T} P F_{1}(d(k))\right]\right\} \xi_{1}(k) \\
= & \xi_{1}^{T}(k)\left[D_{1}+G_{1}(d(k))\right] \xi_{1}(k), \\
\triangle V_{2}(k)= & x^{T}(k) Q_{1} x(k)-x^{T}\left(k-d_{1}\right) Q_{1} x\left(k-d_{1}\right)+x^{T}\left(k-d_{1}\right) Q_{2} x\left(k-d_{1}\right) \\
& -x^{T}(k-d) Q_{2} x(k-d)+x^{T}(k-d) Q_{3} x(k-d)-x^{T}\left(k-d_{2}\right) Q_{3} x\left(k-d_{2}\right) \\
= & \xi_{i}^{T}(k)\left[e_{1}^{T} Q_{1} e_{1}-e_{2}^{T}\left(Q_{1}-Q_{2}\right) e_{2}-e_{4}^{T}\left(Q_{2}-Q_{3}\right) e_{4}-e_{5}^{T} Q_{3} e_{5}\right] \xi_{i}(k) \\
= & \xi_{i}^{T}(k) D_{3} \xi_{i}(k), \quad i=1,2 .
\end{aligned}
$$

Using Lemma 2.1, we can obtain

$$
\begin{aligned}
& \triangle V_{3}(k)=\sum_{i=-d_{1}}^{-1} \sum_{j=k+i+1}^{k} \eta^{T}(j) R_{1} \eta(j)-\sum_{i=-d_{1}}^{-1} \sum_{j=k+i}^{k-1} \eta^{T}(j) R_{1} \eta(j) \\
& =d_{1} \eta^{T}(k) R_{1} \eta(k)-\sum_{i=k-d_{1}}^{k-1} \eta^{T}(i) R_{1} \eta(i) \\
& \leq \xi_{i}^{T}(k)\left[d_{1}\left(e_{0}-e_{1}\right)^{T} R_{1}\left(e_{0}-e_{1}\right)\right] \xi_{i}(k) \\
& +\xi_{i}^{T}(k)\left(e_{1}^{T}, e_{2}^{T}, e_{6}^{T}, e_{10}^{T}\right)\left\{d_{1}\left(Z_{111}+\frac{1}{3} Z_{122}+\frac{1}{5} Z_{133}\right)\right. \\
& \left.+\operatorname{sym}\left[N_{11} \Pi_{1}+N_{12} \Pi_{2}+N_{13} \Pi_{3}\right]\right\}\left(e_{1}^{T}, e_{2}^{T}, e_{6}^{T}, e_{10}^{T}\right)^{T} \xi_{i}(k) \\
& =\xi_{i}^{T}(k)\left[D_{4}+H_{1}\right] \xi_{i}(k), \quad i=1,2, \\
& \Delta V_{4}(k)=\sum_{i=-d}^{-d_{1}-1} \sum_{j=k+i+1}^{k} \eta^{T}(j) R_{2} \eta(j)-\sum_{i=-d}^{-d_{1}-1} \sum_{j=k+i}^{k-1} \eta^{T}(j) R_{2} \eta(j) \\
& =\left(d-d_{1}\right) \eta^{T}(k) R_{2} \eta(k)-\sum_{i=k-d(k)}^{k-d_{1}-1} \eta^{T}(i) R_{2} \eta(i)-\sum_{i=k-d}^{k-d(k)-1} \eta^{T}(i) R_{2} \eta(i) \\
& \leq \xi_{1}^{T}(k)\left[\left(d-d_{1}\right)\left(e_{0}-e_{1}\right)^{T} R_{2}\left(e_{0}-e_{1}\right)\right] \xi_{1}(k)+\xi_{1}^{T}(k)\left(e_{2}^{T}, e_{3}^{T}, e_{7}^{T}, e_{11}^{T}\right)\left\{\left(d(k)-d_{1}\right)\right. \\
& \left.\times\left(Z_{211}+\frac{1}{3} Z_{222}+\frac{1}{5} Z_{233}\right)+\operatorname{sym}\left[N_{21} \Pi_{1}+N_{22} \Pi_{2}+N_{23} \Pi_{3}\right]\right\} \\
& \times\left(e_{2}^{T}, e_{3}^{T}, e_{7}^{T}, e_{11}^{T}\right)^{T} \xi_{1}(k) \\
& +\xi_{1}^{T}(k)\left(e_{3}^{T}, e_{4}^{T}, e_{8}^{T}, e_{12}^{T}\right)\left\{(d-d(k))\left(Z_{311}+\frac{1}{3} Z_{322}+\frac{1}{5} Z_{333}\right)\right. \\
& \left.+\operatorname{sym}\left[N_{31} \Pi_{1}+N_{32} \Pi_{2}+N_{33} \Pi_{3}\right]\right\}\left(e_{3}^{T}, e_{4}^{T}, e_{8}^{T}, e_{12}^{T}\right)^{T} \xi_{1}(k) \\
& =\xi_{1}^{T}(k)\left[D_{5}+G_{3}(d(k))+G_{4}(d(k))\right] \xi_{1}(k),
\end{aligned}
$$




$$
\begin{aligned}
\Delta V_{5}(k)= & \sum_{i=-d_{2}}^{-d-1} \sum_{j=k+i+1}^{k} \eta^{T}(j) R_{3} \eta(j)-\sum_{i=-d_{2}}^{-d-1} \sum_{j=k+i}^{k-1} \eta^{T}(j) R_{3} \eta(j) \\
\leq & \xi_{1}^{T}(k)\left[\left(d_{2}-d\right)\left(e_{0}-e_{1}\right)^{T} R_{3}\left(e_{0}-e_{1}\right)\right] \xi_{1}(k)+\xi_{1}^{T}(k)\left(e_{4}^{T}, e_{5}^{T}, e_{9}^{T}, e_{13}^{T}\right)\left\{\left(d_{2}-d\right)\right. \\
& \left.\times\left(Z_{411}+\frac{1}{3} Z_{422}+\frac{1}{5} Z_{433}\right)+\operatorname{sym}\left[N_{41} \Pi_{1}+N_{42} \Pi_{2}+N_{43} \Pi_{3}\right]\right\} \\
& \times\left(e_{4}^{T}, e_{5}^{T}, e_{9}^{T}, e_{13}^{T}\right)^{T} \xi_{1}(k) \\
= & \xi_{1}^{T}(k)\left[D_{6}+H_{2}\right] \xi_{1}(k) .
\end{aligned}
$$

From (6)-(10) we have $\Delta V(k) \leq \xi_{1}^{T}(k) \Psi_{1}(d(k)) \xi_{1}(k)$. According to (3), we can obtain $\triangle V(k)<0$ for any $\xi_{1}(k) \neq 0$ and $d(k) \in\left[d_{1}, d\right] \cap N$.

Case (ii): $d(k) \in\left[d, d_{2}\right] \cap N$. Similarly, we have

$$
\begin{aligned}
\triangle V_{1}(k)= & \theta^{T}(k+1) P \theta(k+1)-\theta^{T}(k) P \theta(k) \\
= & \xi_{2}^{T}(k)\left\{E_{4}^{T} P E_{4}-E_{3}^{T} P E_{3}+\operatorname{sym}\left[\left(E_{4}-E_{3}\right)^{T} P F_{2}(d(k))\right]\right\} \xi_{2}(k) \\
= & \xi_{2}^{T}(k)\left[D_{2}+G_{2}(d(k))\right] \xi_{2}(k), \\
\Delta V_{4}(k)= & \sum_{i=-d}^{-d_{1}-1} \sum_{j=k+i+1}^{k} \eta^{T}(j) R_{2} \eta(j)-\sum_{i=-d}^{-d_{1}-1} \sum_{j=k+i}^{k-1} \eta^{T}(j) R_{2} \eta(j) \\
\leq & \xi_{2}^{T}(k)\left[\left(d-d_{1}\right)\left(e_{0}-e_{1}\right)^{T} R_{2}\left(e_{0}-e_{1}\right)\right] \xi_{2}(k)+\xi_{2}^{T}(k)\left(e_{2}^{T}, e_{4}^{T}, e_{7}^{T}, e_{11}^{T}\right)\left\{\left(d-d_{1}\right)\right. \\
& \left.\times\left(Z_{511}+\frac{1}{3} Z_{522}+\frac{1}{5} Z_{533}\right)+\operatorname{sym}\left[N_{51} \Pi_{1}+N_{52} \Pi_{2}+N_{53} \Pi_{3}\right]\right\} \\
& \times\left(e_{2}^{T}, e_{4}^{T}, e_{7}^{T}, e_{11}^{T}\right)^{T} \xi_{2}(k) \\
= & \xi_{2}^{T}(k)\left[D_{7}+H_{3}\right] \xi_{2}(k), \\
& -d-1 \\
\Delta V_{5}(k)= & \sum_{i=d_{2}}^{k} \sum_{j=k+i+1}^{k} \eta^{T}(j) R_{3} \eta(j)-\sum_{i=-d_{2}}^{-d-1} \sum_{j=k+i}^{k-1} \eta^{T}(j) R_{3} \eta(j) \\
= & \left(d_{2}-d\right) \eta^{T}(k) R_{3} \eta(k)-\sum_{i=k-d(k)}^{k-d-1} \eta^{T}(i) R_{3} \eta(i)-\sum_{i=k-d_{2}}^{k-d(k)-1} \eta^{T}(i) R_{3} \eta(i) \\
& +\xi_{2}^{T}(k)\left(e_{3}^{T}, e_{5}^{T}, e_{9}^{T}, e_{13}^{T}\right)\left\{\left(d_{2}-d(k)\right)\left(Z_{711}+\frac{1}{3} Z_{722}+\frac{1}{5} Z_{733}\right)\right. \\
\leq & \xi_{2}^{T}(k)\left[\left(d_{2}-d\right)\left(e_{0}-e_{1}\right)^{T} R_{3}\left(e_{0}-e_{1}\right)\right] \xi_{2}(k)+\xi_{2}^{T}(k)\left(e_{4}^{T}, e_{3}^{T}, e_{8}^{T}, e_{12}^{T}\right)\{(d(k)-d) \\
& \left.\times\left(Z_{611}+\frac{1}{3} Z_{622}+\frac{1}{5} Z_{633}\right)+\operatorname{sym}\left[N_{61} \Pi_{1}+N_{62} \Pi_{2}+N_{63} \Pi_{3}\right]\right\} \\
& \times\left(e_{4}^{T}, e_{3}^{T}, e_{8}^{T}, e_{12}^{T}\right)^{T} \xi_{2}(k) \\
& \left.\left.N_{73} \Pi_{3}\right]\right\}\left(e_{3}^{T}, e_{5}^{T}, e_{9}^{T}, e_{13}^{T}\right)^{T} \xi_{2}(k) \\
& \left.(d(k))+G_{6}(d(k))\right] \xi_{2}(k), \\
& \\
& \\
&
\end{aligned}
$$


and $\triangle V(k) \leq \xi_{2}^{T}(k) \Psi_{2}(d(k)) \xi_{2}(k)$, which under condition (4) indicates that $\Delta V(k)$ is negative definite.

Now by combining Case (i) and Case (ii), $\triangle V(k)$ is negative definite, which guarantees that system (1) is asymptotically stable. This completes the proof.

Remark 3 To show the advantage of time delay division for the stability condition without using time delay division, we omitted the proof process and only display the results in Table 1.

\section{Numerical illustrations}

In this section, we present some numerical examples that often appear in the literature to demonstrate the effectiveness and sophistication of the proposed approach.

Example 1 Consider system (1) with the following parameters:

$$
A=\left(\begin{array}{cc}
0.8 & 0 \\
0.05 & 0.9
\end{array}\right), \quad B=\left(\begin{array}{cc}
-0.1 & 0 \\
-0.2 & -0.1
\end{array}\right)
$$

Example 2 Consider system (1), where

$$
A=\left(\begin{array}{cc}
0.7 & 0.1 \\
0.05 & 0.7
\end{array}\right), \quad B=\left(\begin{array}{cc}
-0.1 & 0.1 \\
-0.1 & -0.2
\end{array}\right) \text {. }
$$

The allowable delay upper bound $d_{2}$ can be found for given $d_{1}$ or vice versa. The simulation results are listed in Tables 1 and 2.

Remark 4 In [12] and this paper, the stability conditions are obtained by using the Wirtinger-based integral inequality and the free-matrix-based integral inequality, respectively. We can see from Table 1 that the latter is superior to the former in reducing the conservatism when time delay division is not used, whereas the conservatism of both is the same when time delay division is used. Therefore, on the basis of some processing techniques, such as the time delay division mentioned before, our future research is discussing the relationship between the tightness of inequalities and the conservatism of criteria.

Remark 5 From Table 2 we can see that with the lower bounds $d_{1}=2,5,7,10,20$, the corresponding $d_{2}=13,14,16,19,29$ obtained in [40], $d_{2}=14,17,18,21,31$ obtained by Theorem 1 in [12], and $d_{2}=15,17,18,21,31$ derived by Theorem 3.1 in this paper. Thus the results obtained from Theorem 3.1 in this paper are significantly better than those obtained from the existing methods.

\section{Conclusion}

In this paper, the problem of stability criteria of discrete systems with interval time-varying delays has been investigated. By means of the discrete form of free-matrix-based integral inequality and the time delay division, less conservative criteria than some existing ones are derived due to a tighter estimation of the new inequality. Finally, some illustrative examples are presented to demonstrate the effectiveness of the obtained method. 


\section{Acknowledgements}

This work of D. Liao was supported in part by the National Natural Science Foundation of China under Grant 61771004 Research Fund for International Young Scientists of National Natural Science Foundation of China under Grant 61550110248, Guangxi Natural Science Foundation Project (No. 2017GXNSFBA198179), Basic Ability Promotion Project for Young and Middle-aged Teachers in Universities of Guangxi (No. 2018KY0214), and Sichuan Science and Technology Plan Project (2018GZ0072, 2018JZ0065).

\section{Funding}

This research was partially supported by the National Natural Science Foundation of China under Grant 61771004, Research Fund for International Young Scientists of National Natural Science Foundation of China under Grant 61550110248, Guangxi Natural Science Foundation Project (No. 2017GXNSFBA198179), Basic Ability Promotion Project for Young and Middle-aged Teachers in Universities of Guangxi (No. 2018KY0214), and Sichuan Science and Technology Plan Project (2018GZ0072, 2018JZ0065).

\section{Availability of data and materials}

Not applicable.

\section{Competing interests}

The authors declare that there is no conflict of interest regarding the publication of this paper.

\section{Authors' contributions}

All authors drafted the manuscript, and they read and approved the final version.

\section{Author details}

${ }^{1}$ School of Mathematical Sciences, University of Electronic Science and Technology of China, Chengdu, P.R. China.

${ }^{2}$ School of Mathematical Sciences and Energy Engineering, Hunan Institute of Technology, Hengyang, P.R. China. ${ }^{3}$ School of Automation and Electronic Engineering, Qingdao University of Science and Technology, Qingdao, P.R. China. ${ }^{4}$ School of Information and Software Engineering, University of Electronic Science and Technology of China, Chengdu, P.R. China.

\section{Publisher's Note}

Springer Nature remains neutral with regard to jurisdictional claims in published maps and institutional affiliations.

\section{Received: 10 October 2018 Accepted: 4 February 2019 Published online: 22 March 2019}

\section{References}

1. Zhang, D., Cheng, J., Park, J.H., Cao, J.: Robust $H_{\infty}$ control for nonhomogeneous Markovian jump systems subject to quantized feedback and probabilistic measurements. J. Franklin Inst. 355(15), 6992-7010 (2018)

2. Liu, L., Meng, X.: Optimal harvesting control and dynamics of two-species stochastic model with delays. Adv. Differ. Equ. 2017, 18 (2017)

3. Cheng, J., Chang, X.H., Park, J.H., Li, H., Wang, H.: Fuzzy-model-based $H_{\infty}$ control for discrete-time switched systems with quantized feedback and unreliable links. Inf. Sci. 436-437, 181-196 (2018)

4. Cheng, J., Ahn, C.K., Karimi, H.R., Cao, J., Qi, W.: An event-based asynchronous approach to Markov jump systems with hidden mode detections and missing measurements. IEEE Trans. Syst. Man Cybern. Syst. (2018, in press). https://doi.org/10.1109/TSMC.2018.2866906

5. Cheng, J., Park, J.H., Cao, J., Zhang, D.: Quantized $H_{\infty}$ filtering for switched linear parameter-varying systems with sojourn probabilities and unreliable communication channels. Inf. Sci. 466, 289-302 (2018)

6. Wu, M., Peng, C., Zhang, J., Fei, M., Tian, Y.: Further results on delay-dependent stability criteria of discrete systems with an interval time-varying delay. J. Franklin Inst. 354, 4955-4965 (2017)

7. Xiong, L., Cheng, J., Cao, J., Liu, Z:: Novel inequality with application to improve the stability criterion for dynamical systems with two additive time-varying delays. Appl. Math. Comput. 321, 672-688 (2018)

8. Li, Z., Bai, Y., Huang, C., Cai, Y.: Novel delay-partitioning stabilization approach for networked control system via Wirtinger-based inequalities. ISA Trans. 61, 75-86 (2016)

9. Chen, J., Park, J.H., Xu, S.: Stability analysis of continuous-time systems with time-varying delay using new Lyapunov-Krasovskii functionals. J. Franklin Inst. 355(13), 5957-5967 (2018)

10. Jiang, X., Han, Q., Yu, X.: Stability criteria for linear discrete-time systems with interval-like time-varying delay. In: Proc. of the 2005, American Control Conference, pp. 2817-2822 (2005)

11. Seuret, A., Gouaisbaut, F.: Wirtinger-based integral inequality: application to time-delay systems. Automatica 49, 2860-2866 (2013)

12. Nam, P., Pathirana, P., Trinh, H.: Discrete Wirtinger-based inequality and its application. J. Franklin Inst. 352, 1893-1905 (2015)

13. Zeng, H., He, Y., Wu, M., She, J.: New results on stability analysis for systems with discrete distributed delay. Automatica 60, 189-192 (2015)

14. Zeng, H., He, Y., Wu, M., She, J.: Free-matrix-based integral inequality for stability analysis of systems with time-varying delay. IEEE Trans. Autom. Control 60, 2768-2772 (2015)

15. Hua, C., Wu, S., Guan, X.: New robust stability condition for discrete-time recurrent neural networks with time-varying delays and nonlinear perturbations. Neurocomputing 219, 203-209 (2017)

16. Chen, J., Xu, S., Zhang, B.: Single/multiple integral inequalities with applications to stability analysis of time-delay systems. IEEE Trans. Autom. Control 62, 3488-3493 (2017)

17. Jiao, S., Shen, H., Wei, Y., Huang, X., Wang, Z.: Further results on dissipativity and stability analysis of Markov jump generalized neural networks with time-varying interval delays. Appl. Math. Comput. 336, 338-350 (2018) 
18. Zhu, F., Meng, X., Zhang, T:: Optimal harvesting of a competitive $n$-species stochastic model with delayed diffusions. Math. Biosci. Eng. 16, 1554-1574 (2019)

19. Luo, J., Tian, W., Zhong, S., Shi, K., Wang, W.: Non-fragile asynchronous event-triggered control for uncertain delayed switched neural networks. Nonlinear Anal. Hybrid Syst. 29, 54-73 (2018)

20. Wang, B., Zhang, D., Cheng, J., Park, J.H.: Fuzzy model-based nonfragile control of switched discrete-time systems. Nonlinear Dyn. 93(4), 2461-2471 (2018)

21. Liu, J., Zhang, J.: Note on stability of discrete-time time-varying delay systems. IET Control Theory Appl. 6(2), 335-339 (2012)

22. Shi, K., Tang, Y., Zhong, S., Yin, C., Huang, X., Wang, W.: Nonfragile asynchronous control for uncertain chaotic Lurie network systems with Bernoulli stochastic process. Int. J. Robust Nonlinear Control 28, 1693-1714 (2018)

23. Lee, T., Park, J., Xu, S.: Relaxed conditions for stability of time-varying delay systems. Automatica 75, 11-15 (2017)

24. Wang, J., Shi, K., Huang, Q., Zhong, S., Zhang, D.: Stochastic switched sampled-data control for synchronization of delayed chaotic neural networks with packet dropout. Appl. Math. Comput. 335, 211-230 (2018)

25. Zhang, C., He, Y., Jiang, L., Wu, M., Zeng, H.: Summation inequalities to bounded real lemmas of discrete-time systems with time-varying delay. IEEE Trans. Autom. Control 62(5), 2582-2588 (2017)

26. Zhu, Q: A dual internal model based repetitive control for linear discrete-time systems. IEEE Trans. Autom. Control (2018, in press). https://doi.org/10.1109/TAC.2018.2836023

27. Zeng, H., Teo, K., He, Y., Wang, W.: Sampled-data-based dissipative control of T-S fuzzy systems. Appl. Math. Model. 65 415-427 (2019)

28. Shi, K., Wang, J., Zhong, S., Zhang, X., Liu, Y., Cheng, J.: New reliable nonuniform sampling control for uncertain chaotic neural networks under Markov switching topologies. Appl. Math. Comput. 347, 169-193 (2019)

29. Wu, M., He, Y., She, J., Liu, G.: Delay-dependent criteria for robust stability of time-varying delay systems. Automatica 40(8), 1435-1439 (2004)

30. Feng, T., Meng, X., Liu, L., Gao, S.: Application of inequalities technique to dynamics analysis of a stochastic eco-epidemiology model. J. Inequal. Appl. 2016, 327 (2016)

31. Long, S., Zhong, S.: Improved results for stochastic stabilization of a class of discrete-time singular Markovian jump systems with time-varying delay. Nonlinear Anal. Hybrid Syst. 23, 11-26 (2017)

32. Gao, H., Chen, T.: New results on stability of discrete-time systems with time-varying state delay. IEEE Trans. Autom. Control 52(2), 328-334 (2007)

33. Zhang, B., Xu, S., Zou, Y.: Improved stability criterion and its applications in delayed controller design for discretetime systems. Automatica 44(11), 2963-2967 (2008)

34. Peng, C: Improved delay-dependent stabilisation criteria for discrete systems with a new finite sum inequality. IET Control Theory Appl. 6(3), 448-453 (2012)

35. Zhang, C., He, Y., Jiang, L., Wu, M.: An improved summation inequality to discrete-time systems with time-varying delay. Automatica 74, 10-15 (2016)

36. Zhu, Q., Xu, J., Huang, D., Hu, G.: Iterative learning control design for linear discrete-time systems with multiple high-order internal models. Automatica 62, 65-76 (2015)

37. Xiang, W.: Parameter-memorized Lyapunov functions for discrete-time systems with time-varying parametric uncertainties. Automatica 87, 450-454 (2018)

38. Zhang, S., Meng, X., Zhang, T.: Dynamics analysis and numerical simulations of a stochastic non-autonomous predator-prey system with impulsive effects. Nonlinear Anal. Hybrid Syst. 26, 19-37 (2017)

39. Meng, X., Zhao, S., Feng, T., Zhang, T.: Dynamics of a novel nonlinear stochastic SIS epidemic model with double epidemic hypothesis. J. Math. Anal. Appl. 433(1), 227-242 (2016)

40. Zhang, J., Peng, C., Zheng, M.: Improved results for linear discrete-time systems with an interval time-varying input delay. Int. J. Syst. Sci. 47(2), 492-499 (2016)

41. Chen, J., Park, J., Xu, S.: Stability analysis of discrete-time neural networks with an interval-like time-varying delay. Neurocomputing 329, 248-254 (2019)

42. Shan, Y., Zhong, S., Cui, J., Hou, L., Li, Y.: Improved criteria of delay-dependent stability for discrete-time neural networks with leakage delay. Neurocomputing 266, 409-419 (2017)

43. Kwon, O., Park, M., Park, J., Lee, S., Cha, E.: Stability and stabilization for discrete-time systems with time-varying delays via augmented Lyapunov-Krasovskii functional. J. Franklin Inst. 350, 521-540 (2013)

44. Meng, X., Li, F., Gao, S.: Global analysis and numerical simulations of a novel stochastic eco-epidemiological model with time delay. Appl. Math. Comput. 339, 701-726 (2018)

45. Meng, X., Wang, L., Zhang, T.: Global dynamics analysis of a nonlinear impulsive stochastic chemostat system in a polluted environment. J. Appl. Anal. Comput. 6(3), 865-875 (2016)

46. Zhang, T., Zhang, T., Meng, X.: Stability analysis of a chemostat model with maintenance energy. Appl. Math. Lett. 68, 1-7 (2017)

47. Chang, Z., Meng, X., Zhang, T.: A new way of investigating the asymptotic behaviour of a stochastic SIS system with multiplicative noise. Appl. Math. Lett. 87, 80-86 (2019) 